\title{
Orbital complications of acute rhinosinusitis
}

\author{
Munteanu Gloria Simona', Bertesteanu Serban Vifor Gabriel ${ }^{1,2}$, \\ Mitran Denisa Maria', Condeescu-Cojocarita Mihnea Florin',2, Popescu Bogdan 1,2, \\ Pascu Paula Luiza', Antonie Catrinel Beatrice ${ }^{1,2}$, Nitu Liliana ${ }^{1,2}$, Popescu Radu Cristian², \\ Cirstea Anca', Arjoca Elena', Nicolaescu Alexandru, Grigore Raluca ${ }^{1,2}$ \\ ${ }^{1}$ E.N.T. Head \& Neck Surgery Clinic - "Colțea” Clinical Hospital Bucharest \\ ${ }^{2}$ E.N.T. Head \& Neck Department - "Dr. Carol Davila” \\ University of Medicine and Pharmacy - General Medicine Faculty - Bucharest
}

\begin{abstract}
Introduction. Rhinosinusitis is the infection and inflammation of at least one of the 4 paranasal sinuses (frontal, maxillary, ethmoid and sphenoid). Their correct diagnosis is generally determined by examining the patient clinically and by rhinosinusal endoscopic examination. There are also cases that show complications or continued evolution despite the correct treatment, and then we will use some imaging investigations to find out more about affection(radiography of anterior sinuses of the face, sinus computerized tomography, sinus magnetic resonance imaging). In particular, acute rhinosinusitis should be carefully managed to avoid complications such as the local ones: orbital cellulitis, orbital abscess, osteomyelitis, cavernous sinus thrombosis; and intracranial complications: meningitis, epidural abscess, subdural abscess, cerebral abscess. Materials and methods. There will be presented all orbital complications according to the cases treated in the ENT Clinic of the Coltea Clinical Hospital. Conclusions. Diagnosis of rhinosinusitis is largely clinical and endoscopic. When complications of the condition arise, these should be investigated imagistically to determine their exact extent and to institute the correct treatment as soon as possible. The complications of rhinosinusitis are medical and surgical life-threatening emergencies, which is why in order to diagnose and correct and quickly institute therapy requires a multidisciplinary approach.
\end{abstract}

Key word: complications, rhinosinusitis, cellulitis, abscess

\section{Introduction}

Acute rhinosinusitis is a pathology commonly encountered in medical practice and has most often a viral etiology. Due to predisposing impairment, such as allergic rhinitis or an exaggerated inflammatory response, edema of the nasal mucosa leads to obstruction of the natural ostium of the paranasal sinus drainage, and bacterial suprainfection may occur. (1) Acute bacterial rhinosinusitis has a fulminant onset and develops for less than four weeks. The most commonly involved germs are Streptococcus pneumoniae, Haemophilus influenzae and Moraxella catarrhalis. Although it is more frequently involved in chronic rhinosinusitis, MRSA can also be incriminated in cases of acute rhinosinusitis. (1) (2) Immunocompromised patients, such as diabetics or leukemia patients, may experience fungal rhinosinusitis, a highly aggressive pathology through invasiveness and rapid development. The most common fungal species are Aspergillus and Mucor. (3)

The diagnosis of acute rhinosinusitis is clinically, the patient accusing nasal obstruction, anterior or posterior rhinorrhea, pressure or pain at the anterior 
sinus sites, hyposmia, fever and fatigue. Election investigation is nasal endoscopy and secretion from the middle meatus for cytobacteriological examination. (4) (5) Simple anterior X-ray examination of the face provides little information to establish the diagnosis and is not indicated. Computerized tomography or magnetic resonance imaging are investigations of cases where there are loco-regional complications. (1) (2)

Acute rhinossinusitis may cause complications by invasion of structures adjacent to paranasal sinuses and may be orbital, intra-cranial or bone wall. Dissemination of the infection predisposes to functional eye damage, neurological damage or even death despite appropriate treatment. Mainly, in the case of acute rhinosinusitis complications, the anterior orbital and the anterior skull is affected due to the proximity of the ethmoidal and frontal sinuses to these structures. The mechanism by which the adjacent structures are affected is either by contiguity and the loss of anatomical barriers, or by haematological dissemination. (3)

\section{Materials and Methods}

Periorbital and orbital complications of acute rhinosinusitis are most frequently encountered, followed by intra-cranial complications and bone complications, which are the most rare. In most cases, the infection has ploy-microbial background. (1) Progression of disease from localized sinus infection to orbital complications can be explained using Chandler classification. Class I represents periorbital cellulite, the inflammatory process being located at the periorbital spaces delimited by the orbital septum, without orbital damage, and the patient accuses the palpebral edema without pain in the eye and without affecting eyesight. In Class II Chandler, the inflammatory process goes beyond the orbital septum and affect orbital content - orbital cellulite. Class III represents the subperiosteal abscess when the infectious process is located between the papyracea lamina and the periorbital space. The orbital abscess is Chandler Class IV and cavernous sinus thrombosis is Class V. (Fig 1) (3)

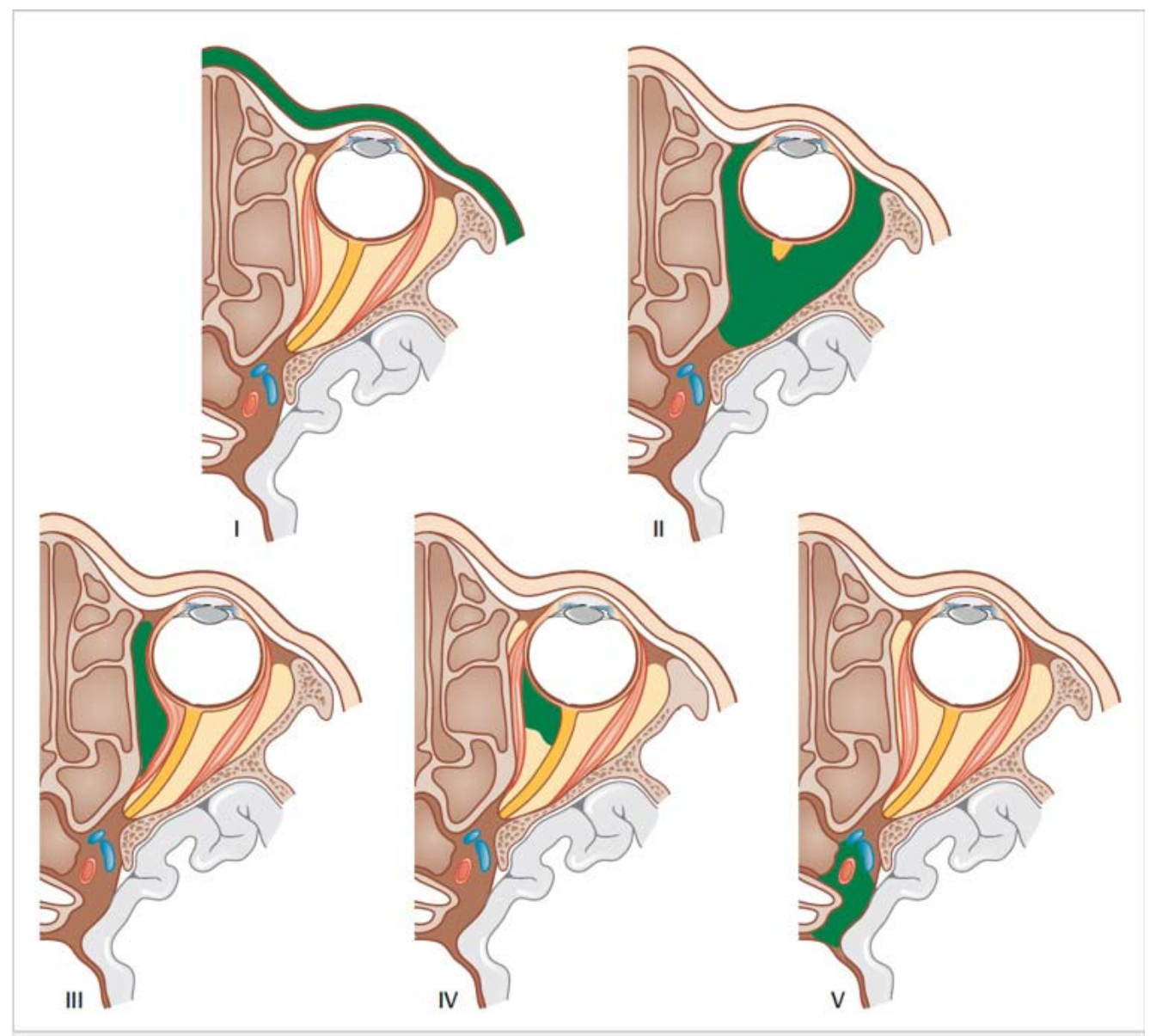

Fig 1. Chandler's classification of periorbital and orbital complications (3) 


\section{Preseptal cellulite}

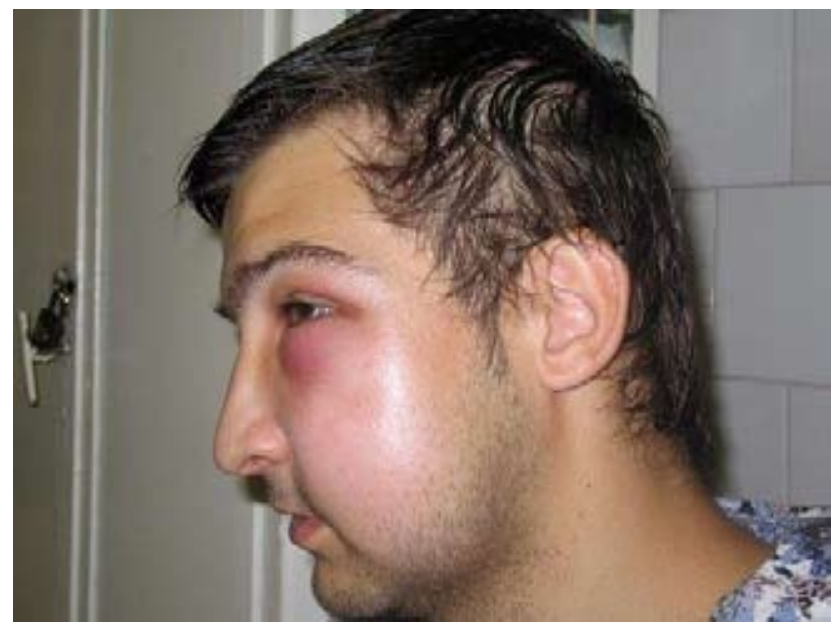

Fig 2. Patient with preseptal cellulite, as a complication of acute rhinosinusitis of the left ethmoidal sinus

Preseptal cellulitis is associated with acute infections of paranasal sinuses. In ethmoiditis the infection spreads through the papyracea lamina (fig 1). In maxillary sinusitis, the infection crosses the orbit floor (Fig 3). In the frontal rhinosinusitis, the infectious process can get into orbit through its ceiling. The infection can dissect the bone causing subperiosteal abscesses. Without proper and rapid treatment, orbital complications lead to blindness and death. (2) (3)

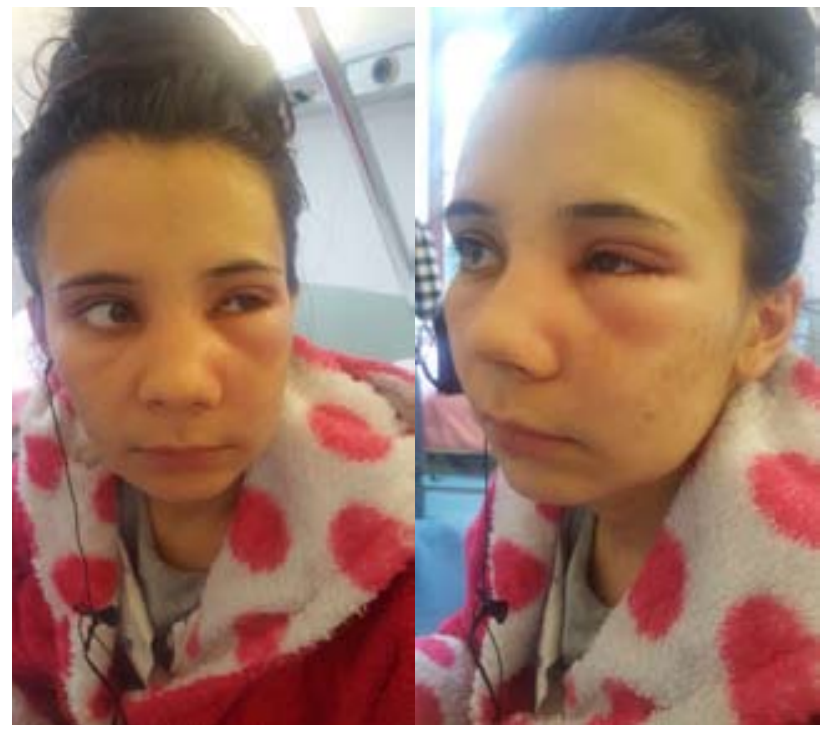

Fig 3. Patient with preseptal cellulite as a complication of acute rhinosinusitis of the left maxillary sinus

Generally, proper antibiotic treatment parenteral or oral is enough to cure the disease. In cases with severe signs and symptoms, broad-spectrum antibiotic therapy and hospitalization are required, followed by appropriate oral antibiotics, as improvements occur. (2)

\section{Orbital cellulite \&}

\section{Subperiosteal abscess}

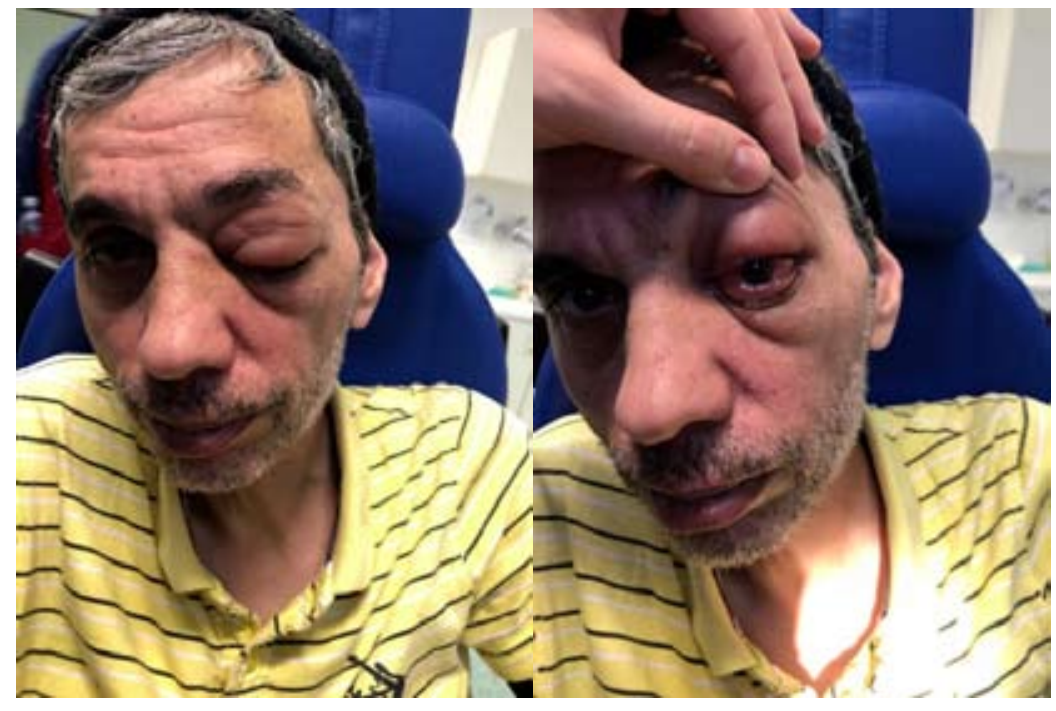

Fig 4. Patient with orbital cellulite and acute frontal rhinosinusitis 


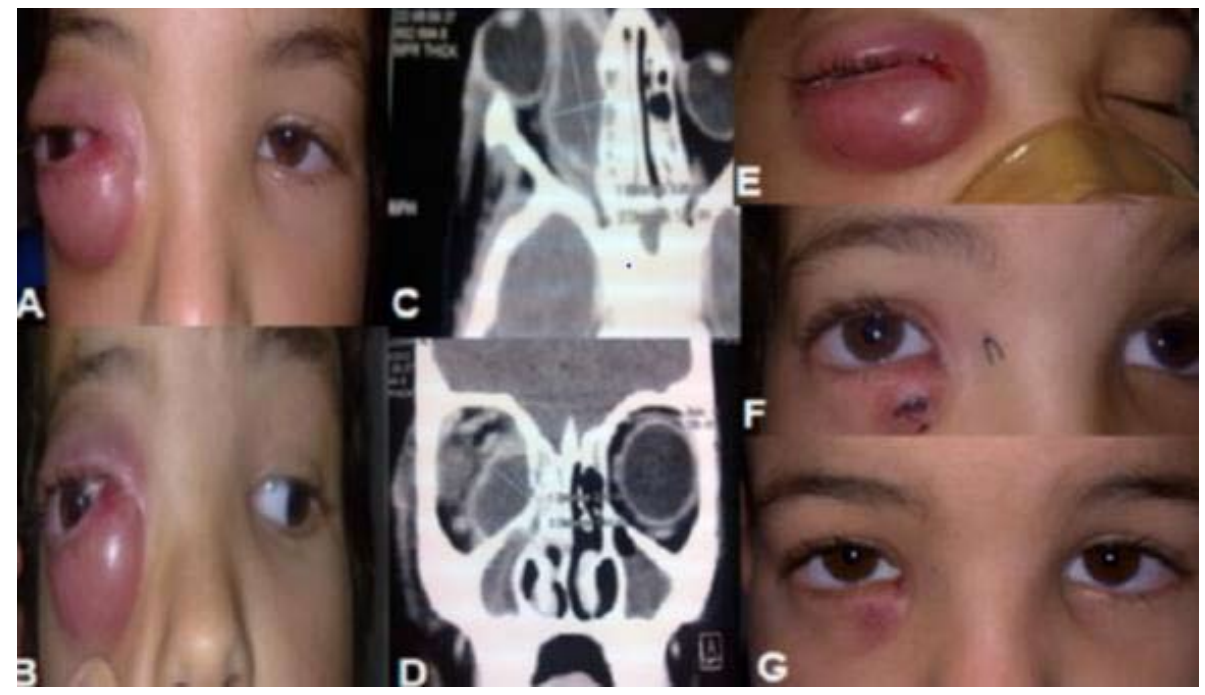

Fig 5. Patient with subperiosteal abscess (Elouarradi H, Daoudi R - Pan Afr Med J (2014))

At the clinical examination, the patient with orbital cellulitis (Fig 4) has edema and erythema of the tissues near orbit. $28.8 \%$ of the orbital cellulite are complications of rhinosinusitis. Examination of the patient is, in general, very painful, and preseptal cellulitis, unlike the subperiosteal abscess (Fig 5), no movement of the eyeball is observed. Axial proptosis is not pathognomonic, requiring differential imaging or surgical diagnosis between orbital cellulitis and subperiosteal abscess. (3)

In the case of orbital cellulite, parenteral treatment is enough. Surgical treatment is indicated when the patient is not responding to treatment, or his condition deteriorates during treatment; when decrease or deterioration of motility or visual acuity occurs; in case of the presence of cranial neuropathies; or if the patient has an abscess, other than a subperiosteal one, small and medial localized. (3) The treatment of the subperiosteal abscess is surgical, endoscopic or open. The open surgery approach ends with postoperative facial scarring, delayed healing, suture infections, overlapping diplopia, recurrent periorbital cellulitis with or without subperiosteal abscess. In the case of postseptal cellulitis, associated with ophthalmoplegia and loss of vision, urgent decompression and drainage of the orbit is immediately required. (2) (3)

\section{Orbital abscess}

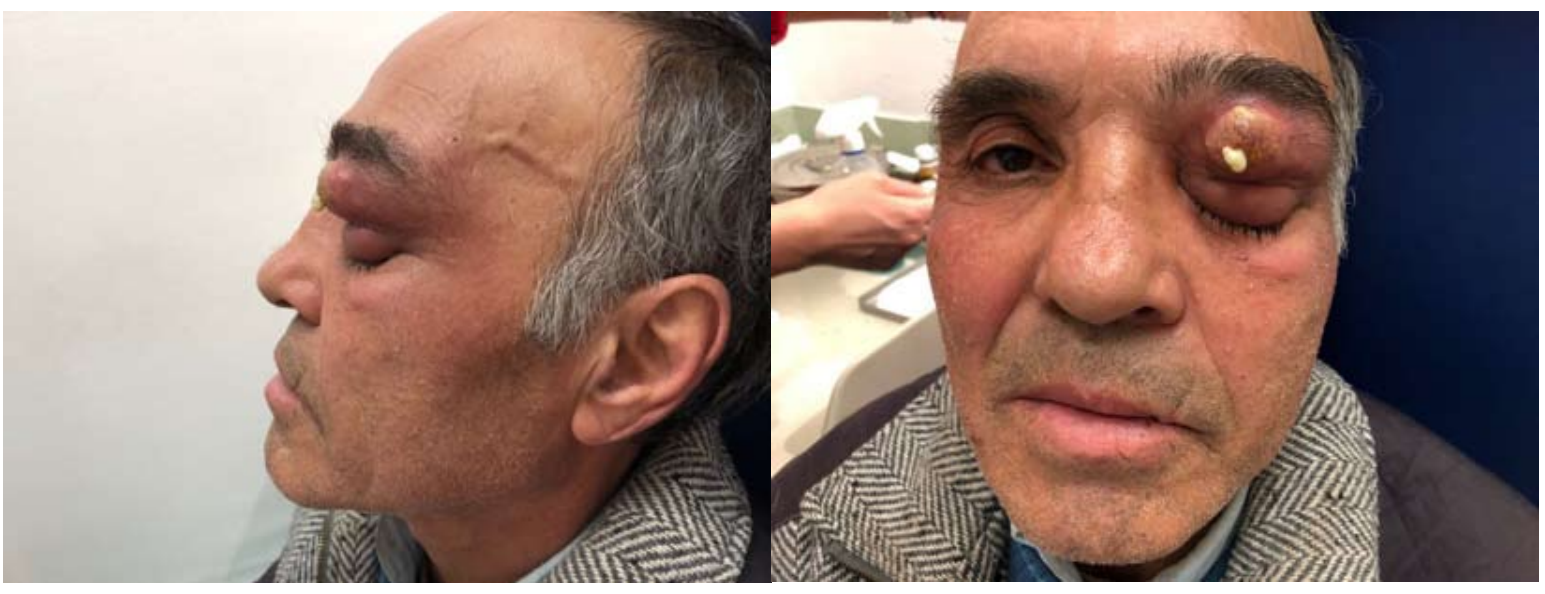

Fig 6. Patient with orbital abscess 
Clinically, the orbital abscess can not be distinguished from orbital cellulite. It can present with severe prooptosis, ophthalmoplegia and displacement of the eye ball. (Fig 5) (3)

\section{Cavernous sinus thrombosis:}

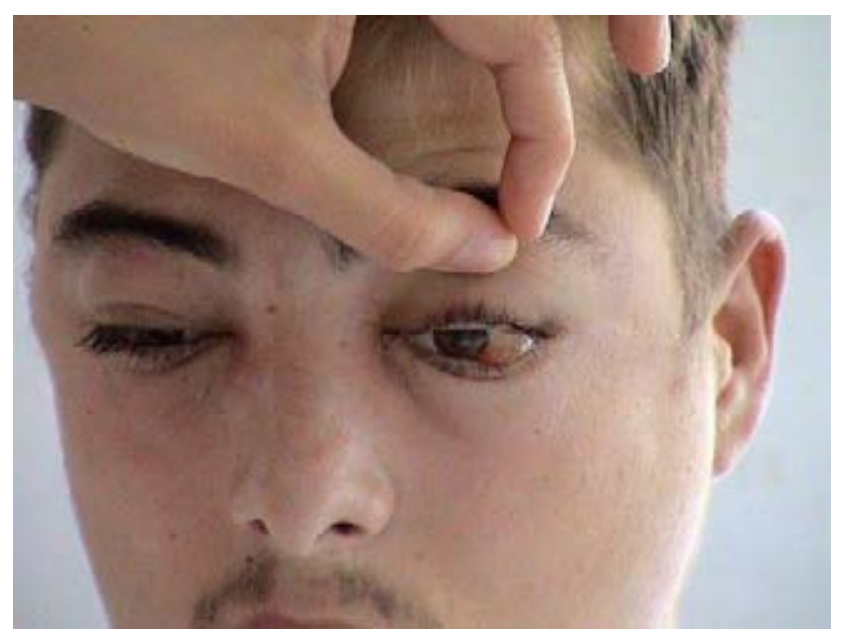

Fig 7. Patient with sinus cavernous thrombosis and chemosis

Cavernous sinus thrombosis is a life-threatening condition. It is produced by acute sphenoid rhinosinusitis and orbital cellulitis. Signs of suspicion are headache, orbital signs, cranial neuropathies. Headache is intense, with the tendency to worsen and does not respond to analgesics. The pain is located at the level of the territory innervated by the branches of the trigeminal nerve and it is unilateral. This affection assosciated diplopia, periorbital edema, mental status alteration, ptosis, photophobia, chemosis, side vision restrictions, midriasis, hypoesthesia or hyperesthesia. (3) (4)
The treatment of cavernous sinus thrombosis requires massive intravenous antibiotic treatment with or without cavernous sinus drainage (dangerous because of the high risk of morbidity). To this two should be added surgical drainage of the primary source of infection (sinuses) and anticoagulation therapy for 4-6 weeks. (3) (4)

\section{Conclusions}

- The most common isolated microorganisms are S. Pneumoniae, Staph Aureus, other streptococcal and anaerobic species.

- Diagnostic delay increases the probability of adverse events.

- The periosteal abscess, and less the postseptal abscess or orbital cellulite, are the most common stages of Chandler; they are very rarely accompanied by orbital abscess and profound sinus thrombosis.

- Endoscopic drainage is preffered in superior or refractory orbital abscesses.

- Preseptal and postseptal cellulitis, small subperiosteal abscesses can be treated with intravenous antibiotics.

- To differentiate the subperiosteal abscess from orbital cellullitis requires imaging investigations or surgery.

- Head CT scan is required in immunocompromised patients with a history of central nervous system disease, recent onset serizures, papillary edema, altered state of consciousness.

- Any delay in initiatind treatment of cavernous sinus thrombosis or orbital abscess may result in blindness, intracranial extension of the infection and death.

\section{Bibliografie}

1. Flint, Paul W, and Charles W. Cummings. Cummings Otolaryngology Head \& Neck Surgery. , 2010

2. Fokkens W.J., Lund V.J, Mullol J., et al - European Position Paper on Rhinosinusitis and Nasal Polyps 2012 , Vol 23, 2012

3. Yen, M.T, Johnson, T.E., et al - Orbital Cellulitis and Periorbital Infections, 2018

4. Peter H Hwang, MD; Zara M Patel, MD. Acute sinusitis and rhinosinusitis on adults: Clinical manifestations, UpToDate, 2017

5. Anniko, M.; Sprekelsen, M.; Bonkowsky, V.; Bradely, P.; Iurato, S.: European Manual of Medicine Otorhinolaryngoloy, Head \& Neck Surgery, Germany, 2010, ISBN 978-3-540-42940-1 